Service social

\title{
La qualité relationnelle dans la famille recomposée : perspectives du parent et de l'enfant
}

\section{Madeleine Granger, Linda Desrochers-Boudreau, Roger Laroche et Richard Cloutier}

Volume 39, numéro 3, 1990

Familles recomposées après divorce

URI : https://id.erudit.org/iderudit/706500ar

DOI : https://doi.org/10.7202/706500ar

Aller au sommaire du numéro

Éditeur(s)

École de service social de l'Université Laval

ISSN

1708-1734 (numérique)

Découvrir la revue

Citer cet article

Granger, M., Desrochers-Boudreau, L., Laroche, R. \& Cloutier, R. (1990). La qualité relationnelle dans la famille recomposée : perspectives du parent et de l'enfant. Service social, 39(3), 50-63. https://doi.org/10.7202/706500ar

\section{Résumé de l'article}

Cette recherche porte sur les facteurs associés à la qualité relationnelle dans la famille recomposée. Elle met en relation l'âge de l'enfant, le genre de son parent biologique, le degré de contact avec le parent non gardien et la situation socio-économique familiale avec deux indicateurs de qualité relationnelle : la perception de la qualité du fonctionnement familial et la satisfaction à l'égard de ce fonctionnement. Le rendement scolaire est aussi considéré en tant que mesure du niveau d'adaptation extrafamiliale de l'enfant. L'échantillon se compose de 45 dyades enfant-parent biologique (17 pères et 28 mères) vivant dans des familles recomposées depuis en moyenne trois ans et huit mois. Aucune des hypothèses n'a trouvé d'appui dans les données issues des perceptions parentales. Deux hypothèses ont été confirmées à partir des perceptions des jeunes : a) les enfants de 9 à 11 ans perçoivent une meilleure qualité relationnelle dans leur famille recomposée que les adolescents de 14 à16 ans; et $b$ ) dans leur nouvelle famille, les enfants vivant avec leur mère biologique sont plus satisfaits des relations familiales que ceux qui vivent avec leur père biologique. Les conséquences pour la pratique et pour la recherche sont définies. 
Madeleine Granger, Linda Desrochers-Boudreau, Roger Laroche, Richard Cloutier, École de psychologie et Centre de recherche sur les services communautaires, Université Laval.

\title{
La qualité relationnelle dans la famille recomposée : perspectives du parent et de l'enfant
}

\author{
Madeleine Granger \\ Linda Desrochers-Boudreau \\ Roger Laroche \\ Richard Cloutier
}

La famille est dite recomposée lorsque l'enfant vit avec l'un de ses parents biologiques et un nouveau parent. Ce nouveau système familial est fondé sur des bases qui lui sont propres et qui diffèrent de celles de la famille nucléaire traditionnelle (Anderson et White, 1988; Kurdek, 1989; Mills, 1984; Papernow, 1984). La complexité et la nouveauté des relations que vivent ces familles en font un ensemble sans frontières bien définies et qui n'a pas encore le statut de vraie famille dans notre société (Blais et Tessier, 1990; Hobart, 1988). Il s'agit cependant d'un phénomène social important, car une forte proportion des enfants qui naissent aujourd'hui connaîtront une recomposition de leur famille avant d'atteindre l'âge de 18 ans (Careau et Cloutier, 1990; Glick, 1984).

\section{La question à l'étude}

La recomposition est un processus qui provoque un nouveau partage des liens familiaux, parce qu'elle se fonde sur des gains et des pertes entre les membres nouveaux ou anciens. La recomposition provoque aussi une remise en question des rôles attendus de chacun dans les rapports mutuels actuels et futurs (Ganong et Coleman, 1986; 
Roberts et Price, 1989; Sauer et Fine, 1988). Cette dynamique de remise en question des frontières interpersonnelles et des rôles familiaux prend place dans un contexte humain et matériel qui peut se révéler favorable ou défavorable à la réussite de l'ajustement mutuel. Quels sont les facteurs qui sont associés à la perception de la réussite de l'ajustement relationnel dans la famille recomposée ? C'est sur cette question que porte la présente étude en mettant à contribution la perception réciproque de parents biologiques et de leur enfant quant à l'ajustement relationnel qui prévaut dans leur famille recomposée.

Quatre facteurs jugés importants par les études sur le sujet ont été retenus ici. Ce sont le sexe du parent gardien et de son enfant (la variable "genre "), l'âge de l'enfant, le degré de contact avec le parent non gardien (tel que mesuré par le temps de contact effectif) et le niveau socio-économique de la nouvelle famille. Chez les parents et les enfants ces variables ont été mises en relation avec la perception de la qualité du fonctionnement familial et de la satisfaction à l'égard de ce fonctionnement. Le rendement scolaire de l'enfant a aussi été considéré à titre d'indice psychosocial du jeune.

\section{Prédicteurs de la qualité relationnelle de la famille recomposée}

Âge et genre. Le type de relation qui s'établit entre les membres de la famille varierait selon l'âge et le genre des enfants résidant dans cette nouvelle famille ainsi que selon le genre du parent gardien. Knaub, Hanna et Stinnet (1984) affirment que les enfants en bas âge seront plus conciliants envers un nouveau parent, quel que soit son genre. Wahler et Dumas (1989) observent que l'indice de détresse psychologique des mères de familles intactes diminue à mesure que l'enfant grandit et demande moins d'interventions de contrôle de la part du parent. Kurdek (1990) observe la même tendance auprès de 66 familles recomposées matricentriques. Dans chacun de ces cas, c'est la charge d'enfants de moins de six ans qui est perçue comme la plus lourde par les mères, les pères n'affichant pas de différence.

En revanche, d'autres travaux observent qu'à l'approche de l'adolescence, les relations avec le nouveau parent (père ou mère) peuvent devenir plus difficiles : les problèmes deviennent plus aigus parce que les adolescents veulent plus de pouvoir et sont plus provocants à l'égard des parents ou des nouveaux parents (Amato, 1989, 1990; Cloutier, 1982; Hobart, 1988; Hetherington, Stanley-Hagan et Anderson, 1989; Pink et Wampler, 1985).

Il importe de signaler ici que les méthodes de collecte de données varient d'une étude à l'autre et que la perception des enfants n'est à peu près jamais prise en considération même si l'on s'accorde 
à dire que les jeunes peuvent influencer significativement la qualité relationnelle dans la famille, qu'elle soit intacte ou réorganisée.

En ce qui a trait au genre, des études suggèrent que les garçons vivraient des relations familiales plus harmonieuses que les filles dans les familles recomposées, notamment à cause d'une meilleure acceptation du nouveau parent (Hobart, 1988; Brand et Clingempeel, 1987; Clingempeel, Brand et levoli, 1984). Les garçons développeraient une relation de camaraderie avec leur père et la nouvelle mère apporterait un soutien accru à cette relation. La fille, quant à elle, établirait une relation privilégiée avec sa mère durant la période monoparentale et craindrait que l'arrivée du nouveau père ne brise cette relation (Hobart, 1988).

Lorsque la fille demeure avec son père, $c^{\prime}$ est généralement que leur relation est très proche et que la relation avec sa mère est plus distante (Clingempeel et al., 1984). Cloutier, Careau et Drolet (1988) observent cependant que la garde au père est souvent une formule qui remplace la garde à la mère, au moment où cette dernière ne peut plus assumer cette responsabilité. Les filles exerceraient alors certains rôles avec lesquels la nouvelle mère entrerait en compétition (soutien émotionnel, confidente, aide pour l'entretien ménager...). Le fait que les filles et les garçons semblent entretenir des relations différentes avec leur parent biologique durant la période monoparentale pourrait expliquer, au moins en partie, les différences dans les relations avec un nouveau parent.

Contacts avec le parent non gardien. Plusieurs auteurs consultés affirment qu'une fréquence modérée de contacts entre l'enfant et son parent biologique non gardien favorisent de meilleures relations familiales (Brand et Clingempeel, 1987; Clingempeel et Segal, 1986). Clingempeel et Brand (1985) parlent même d'un lien curvilinéaire entre la fréquence des contacts et la qualité des relations : des contacts modérés favoriseraient une meilleure adaptation, d'où une qualité relationnelle plus élevée. De même, des contacts trop fréquents avec le parent non gardien. augmentent l'ambiguïté du rôle du nouveau parent et empêchent le développement de la cohésion familiale, tandis que des contacts trop peu fréquents nuisent à l'établissement d'une bonne relation puisque l'enfant craint que le nouveau parent remplace son parent biologique. Récemment, Visher et Visher (1990) ont cependant souligné l'importance d'une coalition entre parents biologiques (donc, ex-conjoints) dans le succès de la famille recomposée : un contexte de coopération est indispensable pour que l'enfant surmonte ses conflits de loyauté dans ses relations avec ses parents et ses nouveaux parents.

Situation socio-économique. Un autre facteur potentiellement important pour l'établissement de bonnes relations interpersonnelles 
est le niveau de scolarité du parent gardien, facteur relié à la situation socio-économique du milieu familial. Selon White (1979), la scolarité parentale s'avère être le meilleur prédicteur du succès du remariage et du bonheur conjugal. Ce dernier auteur précise que ce sont surtout des facteurs économiques qui incitent les femmes à se remarier si elles ont de jeunes enfants, tandis que les hommes qui ont les moyens de faire vivre deux familles se remarieront parce qu'ils le désirent et non par nécessité. Cette différence motivationnelle reliée au statut socio-économique favorise les hommes qui s'avouent être " au moins aussi heureux " dans leur remariage qu'à leur premier mariage (Demaris, 1984; White et Booth, 1985), tandis que les femmes se considèrent "moins heureuses" qu'à leur premier mariage (White, 1979).

\section{Stratégie générale de l'étude}

Les relations entre les membres constituent les matériaux premiers de la famille. L'attachement parent-enfant, l'influence éducative de la famille, le style parental, la transmission intergénérationnelle, etc., sont autant de réalités essentiellement relationnelles, c'est-à-dire fondées sur l'interaction entre deux ou plusieurs membres d'une famille, avec l'influence réciproque exercée par chacun (Hinde et Stevenson-Hinde, 1988). L'appréciation mutuelle de la relation qu'entretiennent le parent et l'enfant constitue la variable dépendante de la présente étude. À l'instar de nombreux travaux déjà publiés, la représentation que chacun se fait des relations familiales y est utilisée comme base de l'évaluation de l'ajustement familial (Hinde et Stevenson-Hinde, 1988; Olson et McCubbin, 1983; Roberts et Magrab, 1991). La notion de qualité relationnelle renvoie donc ici à l'appréciation que fait l'enfant ou le parent des interactions qu'il vit dans sa famille recomposée. Dans les écrits sur le sujet, plusieurs stratégies ont été utilisées pour mesurer la qualité relationnelle ou des concepts associés (ex. : qualité de la communication). Ici, la qualité relationnelle sera mesurée selon deux indicateurs : 1) la satisfaction perçue à l'égard de la vie familiale dans son ensemble; 2) l'équilibre perçu du fonctionnement familial. Ces indices familiaux seront obtenus auprès de l'enfant et du parent biologique avec lequel il vit dans sa famille recomposée.

Une satisfaction élevée à l'égard du fonctionnement familial indique qu'un individu perçoit ses attentes et ses besoins comme comblés dans sa famille (Olson, Russel et Sprenkle, 1983). Selon Cloutier et Groleau (1987) la satisfaction a souvent servi à mesurer la qualité relationnelle perçue. Quant à l'équilibre perçu du fonctionnement familial, il sera évalué à partir du modèle circomplexe de Olson et al. (1985). Selon ce modèle, une famille est équilibrée lorsqu'elle a des 
niveaux modérés de cohésion et d'adaptabilité. Une cohésion très faible indique une séparation ou un désengagement des membres de la famille, tandis qu'une cohésion très élevée signifie la symbiose entre eux. Une absence d'adaptabilité indique une rigidité, tandis qu'une adaptabilité très élevée indique beaucoup d'imprévisibilité dans le fonctionnement familial.

À l'instar de plusieurs autres travaux, le rendement scolaire de l'enfant a été utilisé ici comme reflet de l'ajustement social et affectif de l'enfant. En effet, cette variable extrafamiliale a souvent été reconnue comme un reflet sensible de l'adaptation fonctionnelle de l'enfant dans son milieu de vie (Cloutier, 1985; Demo et Acock, 1988; Dolto, 1988; Smith, 1990). Dans la présente étude les moyennes en français et en mathématiques au cours du dernier trimestre serviront de mesure du rendement scolaire.

Quatre hypothèses sont formulées à la lumière des tendances précédemment relevées dans la littérature. La première hypothèse porte sur la relation entre la qualité relationnelle et le facteur genre du parent et de l'enfant; chaque volet de cette hypothèse fera l'objet d'une vérification spécifique : a) la qualité relationnelle est plus élevée chez les garçons que chez les filles; $b$ ) la qualité relationnelle est plus élevée lorsque la mère biologique a la garde plutôt que lorsque c'est le père qui l'assume. La deuxième hypothèse propose l'existence d'un lien significatif entre la qualité relationnelle et l'âge de l'enfant. Selon cette hypothèse, la qualité relationnelle serait plus élevée chez les enfants de 9 à 11 ans que chez les adolescents de 14 à 16 ans. La troisième hypothèse suppose l'existence $d^{\prime}$ 'une relation curvilinéaire entre la qualité relationnelle et le nombre de jours par semaine passés avec le parent non gardien. Cela signifie qu'aux deux extrêmes de la courbe, on observerait une qualité relationnelle moindre. La quatrième hypothèse propose l'existence $d^{\prime}$ un lien positif entre la qualité relationnelle et le niveau socio-économique de la famille reflété par a) le revenu familial global et $b$ ) le niveau de scolarité du parent gardien.

\section{Méthode}

Sujet. L'échantillon comprend 45 dyades enfant/parent biologique qui sont réparties de la façon illustrée au tableau qui suit. Comme dans la population, cet échantillon compte plus de mères qui ont la garde de leurs enfants que de pères. Lorsque les deux parents d'un enfant vivaient en famille recomposée, la famille dans laquelle l'enfant passe le plus de jours par semaine a été choisie. En cas d'égalité, la famille du père biologique a été retenue, de façon à agrandir 
cette catégorie déjà petite. Cet échantillon regroupe donc 18 enfants qui fréquentent l'école primaire et 27 qui sont au secondaire. En moyenne, les familles sont recomposées depuis 3 ans et 8 mois, et les enfants $y$ vivent 5,5 jours par semaine. Le revenu familial moyen se situe à environ 35000 \$ et un peu plus de la moitié des parents biologiques possèdent une scolarité universitaire, proportion plus élevée que celle de l'ensemble de la population. II faut noter, au bas du tableau qui suit, la différence importante entre le revenu familial moyen des familles patricentriques comparativement à celui des familles matricentriques. La distribution de la scolarité indique aussi que les familles patricentriques comptent une proportion plus élevée de parents ayant suivi un cours collégial ou universitaire.

\section{Répartition des dyades selon l'âge et le genre de l'enfant ainsi que selon le genre de son parent biologique}

\begin{tabular}{|lcccc|}
\hline & & Mères & Pères & Total \\
\hline Filles & $9-11$ ans & 6 & 5 & 11 \\
\multirow{2}{*}{ Garçons } & $14-16$ ans & 9 & 5 & 14 \\
\multirow{2}{*}{ Total } & $9-11$ ans & 5 & 2 & 7 \\
& $14-16$ ans & 8 & 5 & 13 \\
Revenu familial moyen & 28 & 17 & 45 \\
\hline \multirow{2}{*}{ Scolarité du } & Université & 10 & 13 & 23 \\
parent répondant & Collégial & 1 & 3 & 3 \\
& Secondaire & 16 & 1 & 17 \\
\multirow{2}{*}{ Total } & Primaire & 1 & 0 & 1 \\
\hline
\end{tabular}

Instruments de mesure. Deux échelles standardisées ont été utilisées et administrées à la fois au parent biologique et à l'enfant cible. Ces échelles ont été traduites par Cloutier et Groleau (1987).

Instrument 1 : Échelle de fonctionnement familial

L'échelle " Family Adaptability and Cohesion Scales » (FACES III), tirée du «Family Inventories » de Olson et al. (1985), mesure les degrés de cohésion et d'adaptabilité de la famille tels qu'ils sont perçus par les membres. Elle comprend 20 items de type Likert et permet, grâce au calcul du "Distance From Center »(DFC), de mesurer le 
niveau d'équilibre de la vie familiale en vertu du modèle circomplexe de Olson. Le DFC permet en effet d'incorporer simultanément les résultats de cohésion et d'adaptabilité sur une mesure linéaire (OIson et al., 1985). La version anglophone du test a une validité de contenu bien établie par les travaux de Olson et al. $(1983 ; 1985)$. Elle discrimine bien entre les familles fonctionnelles et celles éprouvant des problèmes et sa relation est faible avec la désirabilité sociale. Dans la version américaine, la consistance interne s'élève à 0,68 et la fidélité test-retest varie de 0,80 à 0,83 à quatre semaines d'intervalle (normalisation effectuée auprès de 2865 sujets). La version française de l'échelle a été administrée à 133 sujets (Cloutier et al., 1988; Careau et Cloutier, 1990) et a permis d'obtenir un coefficient de consistance interne variant entre 0,78 et 0,86 selon que les répondants étaient des pères ou des mères de famille, ce qui est considéré comme bon (Nunnaly, 1967).

Instrument 2 : Échelle de satisfaction familiale

Cette échelle tirée du "Family Inventories " (Olson et al., 1985) mesure le degré de satisfaction concernant le fonctionnement familial. Elle comprend 14 items de type Likert. Dans la version originale, l'alpha de Cronbach donne une valeur de 0,92 pour la consistance interne et la corrélation de Pearson donne une fidélité test-retest de 0,75 à cinq semaines d'intervalle (normalisation effectuée auprès de 2462 personnes). L'administration de la version française à 310 sujets (Cloutier et al., 1988; Careau et Cloutier, 1990) a permis d'obtenir un alpha de Cronbach variant de 0,82 à 0,91 selon qu'il s'agissait de répondants enfants, mères ou pères dans la famille. Cinq variables indépendantes ont servi à vérifier les hypothèses : 1) le genre de l'enfant et de son parent; 2) l'âge de l'enfant divisé en deux catégories, 9 à 11 ans et 14 à 16 ans; 3) l'horaire de garde partagé en trois groupes selon que l'enfant passe 2-4 jours, 5-6 jours ou 7 jours en moyenne par semaine avec son parent biologique; 4) la scolarité du parent gardien; et 5) le revenu familial total.

La collecte de données a principalement été effectuée à l'aide de questionnaires écrits. Ces questionnaires, les deux échelles standardisées de Olson, ont été remplis à la maison et retournés par la poste. Les autres données, le revenu familial, l'horaire de garde et le rendement scolaire, ont été recueillies par entrevue téléphonique. Les moyennes en mathématiques et en français ont été vérifiées auprès des enseignants. Une récompense symbolique de $8,00 \$$ était remise à chaque répondant (parent et enfant) lorsqu'il avait rempli le questionnaire. 


\section{Résultats et discussion}

À titre indicatif sur le plan métrique, une analyse des relations entre les deux échelles de qualité relationnelle (DFC et satisfaction) et le rendement scolaire a d'abord été effectuée au moyen de corrélations de Pearson. Cette analyse a permis d'établir les liens entre les perceptions des parents et celles des enfants. Aucune relation significative n'a été observée entre les deux indicateurs (DFC et satisfaction) provenant des parents $(r=0,01)$. Pour les données provenant de l'enfant, une relation significative a été trouvée entre l'indice de satisfaction et le DFC $(r=0,52, p<0,0001)$. En ce qui a trait au rendement scolaire en français, il avait une relation significative avec le DFC de l'enfant $(r=0,35, p<0,0001)$, indiquant un lien entre le rendement scolaire et l'ajustement familial perçu. Lorsque les données de l'enfant sont mises en relation avec celles du parent, aucune corrélation ne se révèle significative entre les indices. Olson et al. (1985) ont obtenu des résultats semblables lorsqu'ils comparaient les résultats du DFC entre différents membres de la famille. Selon eux, de telles différences se retrouvent souvent dans les études où l'on compare des perceptions de membres d'une même famille.

$\mathrm{Au}$ regard des hypothèses, aucune tendance significative n'est ressortie de l'analyse des données provenant des parents. Seules les données obtenues auprès des jeunes révèlent des tendances significatives. Donc, si cette étude n'avait tenu compte que des perceptions parentales, aucune de nos hypothèses n'aurait trouvé d'appui. Les données de Cloutier et Groleau (1987) soutiennent cette tendance voulant que les données obtenues auprès des jeunes traduisent parfois une plus grande différenciation que celles issues de leurs parents. La sensibilité parentale plus grande à la désirabilité sociale constitue une explication possible à ce phénomène. Dans le cas qui nous occupe, cependant, il est plausible que le fait d'avoir regroupé sous la rubrique " réponses des parents " les perceptions des pères et celles des mères participe à l'absence de tendances significatives relativement aux hypothèses : il aurait été préférable de disposer d'un échantillon suffisamment vaste pour permettre de séparer les perceptions des mères de celles des pères.

Quant à la première hypothèse proposant une relation entre la qualité relationnelle et le facteur genre (sexe du parent et de l'enfant), les résultats provenant des données des parents et des enfants infirment le premier volet voulant que la qualité relationnelle soit plus élevée chez les garçons que chez les filles. Seuls les résultats provenant des enfants ont permis de confirmer une partie du deuxième aspect de cette hypothèse, à savoir que la satisfaction des enfants 
des deux sexes est plus élevée lorsque c'est la mère biologique qui assume la garde plutôt que le père $(F(1,34)=6,6, p<0,01)$.

Partiellement au moins, ce résultat pourrait s'expliquer par le fait que la garde au père représente souvent une formule de remplacement à la suite d'une incapacité ou d'un refus de la mère de continuer d'assumer cette responsabilité (Cloutier et Groleau, 1988; GilesSims et Urwin, 1989). Sorensen et Goldman (1990) soulignent l'importance, dans le processus d'adjudication et d'appréciation de la garde, de bien prendre en considération les motivations parentales en jeu. Ainsi, la relation parent-enfant dans la famille recomposée patricentrique qui a la garde parce que la mère n'a pas pu continuer à l'assumer ne peut pas être comparée à la relation qui a cours dans le même type de famille où le père a toutefois réclamé la garde dès le moment de la séparation. Dans le présent échantillon, plusieurs de nos familles patricentriques avaient la garde en remplacement d'une garde à la mère. De plus, la garde à la mère est souvent associée à un manque de contact entre l'enfant et son père. La présence masculine qu'offre le beau-père serait alors favorable à l'enfant (Cloutier et Groleau, 1987, Giles-Sims et Urwin, 1989; Sorensen et Goldman, 1990).

La deuxième hypothèse suggérant que la qualité relationnelle perçue par les parents et les enfants est plus élevée dans le groupe des enfants de 9 à 11 ans est confirmée par les résultats provenant des enfants, tant sur le plan de la satisfaction $(t(36)=2,30, p<0,05)$ que sur celui de la perception du fonctionnement familial $(\mathrm{t}(36)=2,50$, $p<0,05)$. Les adolescents ont donc une image moins positive des relations dans leur famille recomposée : moins de cohésion et d'adaptabilité et moins de satisfaction. Ces résultats vont dans le même sens que ceux obtenus par Knaub et al. (1984), Hobart (1988) et Pink et Wampler (1985).

Le rendement scolaire, utilisé ici comme indicateur d'ajustement psychosocial de l'enfant (Demo et Acock, 1988), appuie cette hypothèse $d^{\prime}$ 'un meilleur ajustement des plus jeunes, puisqu'il est plus élevé en français $(\mathrm{t}(42)=4,61, \mathrm{p}<0,0001)$ et en mathématiques $(\mathrm{t}(40,6)=4,10, \mathrm{p}<0,0001)$ chez les 9 à 11 ans que chez les adolescents (14 à 16 ans). II reste à savoir dans quelle mesure cet effet d'âge est relié à la recomposition familiale comme telle et non pas à une baisse typiquement observée chez l'ensemble des jeunes au moment de la transition adolescente.

Vraisemblablement, le redécoupage relationnel et territorial typique de l'adolescence entrerait ici en interaction avec le processus de redéfinition des rôles et des frontières dans la famille recomposée pour augmenter les tensions perçues par les adolescents. La comparaison avec un groupe-témoin de jeunes issus de familles intactes permettrait de voir si cette tendance est le propre des familles recom- 
posées ou si elle existe aussi dans les familles intactes. Dans une étude portant sur 206 familles intactes, Groleau (1987) n'obtenait pas de différence dans le niveau de satisfaction perçu à l'égard de la relation parent-adolescent ou adolescente de 12 à 18 ans.

La troisième hypothèse proposant l'existence $d^{\prime}$ 'une relation curvilinéaire entre la qualité relationnelle et le nombre de jours par semaine passés avec le parent non gardien n'est pas confirmée par nos résultats. Ainsi, la tendance relevée dans la littérature selon laquelle l'absence de relation avec le parent non gardien, de la même façon qu'un contact très soutenu avec lui, affecterait négativement la qualité relationnelle dans la nouvelle famille (Clingempeel et Segal, 1986) ne trouve pas d'appui ici : le temps de contact avec le parent non gardien n'est pas relié significativement à la qualité relationnelle perçue par le jeune dans sa nouvelle famille. La dimension réduite de notre échantillon peut avoir créé une confusion de variables dans le contexte de cette hypothèse. En effet, notre échantillon n'était pas assez grand pour que nous puissions distinguer les familles matricentriques $(N=28)$ des familles patricentriques $(N=17)$ dans la mise en relation du temps de contact avec le parent-visiteur et les indices relationnels; en conséquence, des familles patricentriques affichant un indice de qualité relationnelle moindre ont été regroupées avec des familles matricentriques, ce qui a pu voiler l'effet propre du temps de contact de l'enfant avec son parent non gardien sur la qualité relationnelle elle-même.

La quatrième hypothèse stipulant l'existence d'un lien positif entre la qualité relationnelle perçue et le niveau socio-économique de la famille est infirmée par les données provenant des enfants, c'est-àdire que les enfants issus de familles recomposées financièrement plus à l'aise ne présentent pas des indices plus élevés de qualité relationnelle. Encore ici, la dimension réduite de notre échantillon peut être en cause : les familles recomposées patricentriques sont plus riches et en même temps, ainsi qu'il est révélé par la confirmation de la première hypothèse, les enfants y perçoivent une qualité relationnelle moindre, ce qui peut annuler l'effet positif du niveau socio-économique.

Quant au rendement scolaire, indice que nous avons retenu comme témoin de l'ajustement extrafamilial de l'enfant, nos données indiquent que les parents les plus scolarisés ont des enfants qui réussissent significativement mieux en français $(t(39)=3,6, p<0,001)$ et en mathématiques $(t(38)=4,6, p<0,0001)$. Cette tendance appuie le constat général voulant que des parents plus instruits valorisent davantage le succès scolaire et, par le fait même, incitent leurs enfants à mieux réussir à ce chapitre (Cloutier et Renaud, 1990). Une relation modérée mais significative se retrouve aussi entre un revenu familial 
plus élevé et les résultats scolaires supérieurs de l'enfant en mathématiques $(r=0,34, p<0,05)$. La relation entre le revenu et la scolarité parentale pourrait expliquer cette tendance : les familles à revenu plus élevé valoriseraient davantage la réussite scolaire de l'enfant.

\section{Conclusion}

L'étude précédemment décrite possède sans contredit un caractère exploratoire et elle laisse plusieurs questions sans réponse quant aux facteurs associés à la qualité relationnelle dans la famille recomposée. En mettant à profit aussi bien les hypothèses qu'elle a confirmées que celles qu'elle n'a pas pu confirmer, la démarche permet de définir certaines incidences pratiques ainsi que certaines avenues pour la recherche.

\section{Incidences pratiques}

- La perception des enfants est une source sensible d'indications quant au fonctionnement relationnel dans la famille recomposée et elle fournit une image qui peut se révéler différente de celle obtenue des parents. Cette observation est conforme à ce que plusieurs autres études ont mis en évidence et souligne l'importance d'offrir un droit de parole à l'enfant dans les évaluations et les décisions familiales qui le concernent (Amato, 1990; Barry, 1988; Barry et al., 1985; Cloutier et Groleau, 1987; Jessop, 1981; Moos et Moos, 1986; Olson et McCubbin, 1983);

- l'adolescence, dans la perspective des jeunes tout au moins, semble augmenter le poids du processus de redéfinition des frontières relationnelles et des rôles auquel doivent faire face les membres de la famille recomposée. Cette observation souligne l'importance de tenir compte du statut développemental du jeune dans l'évaluation de la famille recomposée. Un certain nombre de travaux ont déjà montré que la plus grande dépendance de l'enfant à l'égard de ses parents faisait du soutien parental (chaleur, sensibilité, appui, etc.) le déterminant le plus important de la qualité de la relation parent-enfant, tandis que la recherche d'autonomie de l'adolescent ou de l'adolescente faisait du style de contrôle parental (style d'autorité et de supervision, respect de l'intimité personnelle, degré de délégation, de responsabilisation et de confiance, etc.) l'un des déterminants les plus importants de la qualité de la relation parent-adolescent ou adolescente (Amato, 1989; 1990). Sachant que cette relation est reconnue comme fragile dans les familles intactes, elle doit faire 
l'objet d'une attention particulière dans la famille recomposée où le nouveau parent peut assumer prématurément un rôle de contrôle auprès d'un jeune qui n'accepte pas ce nouvel acteur dans ce rôle (Hetherington et al., 1989).

\section{Avenues pour la recherche}

Les limites rencontrées dans la démarche empirique qui précède nous amènent à formuler les observations suivantes :

- il n'est pas souhaitable de tenter de composer une perspective parentale unique constituée d'un regroupement de perceptions paternelles et maternelles, puisque le genre du parent est susceptible d'être un facteur d'influence significatif de la teneur de l'appréciation parentale;

- dans l'évaluation de la qualité relationnelle entre parents et enfants dans la famille recomposée, il importe de tenir compte de l'origine de la garde de l'enfant, car il est fort plausible qu'une garde de remplacement, qui n'a pas vraiment été choisie ni par le parent biologique ni par les enfants, affecte à la baisse la qualité relationnelle qui y prévaut;

- dans le contexte de l'évolution rapide des pratiques familiales en matière de partage des responsabilités parentales après la séparation, un vif besoin ressort de données issues d'échantillons plus vastes de familles recomposées afin de dépasser le niveau exploratoire constamment restrictif quant à la portée généralisable des tendances.

\section{Références bibliographiques}

AMATO, P.R. (1990) "Dimensions of the family environment as perceived by children : A multidimensional scaling analysis », Journal of Marriage and the Family, 52, 613-620.

AмATO, P.R. (1989). « Family processes and the competence of adolescents and primary school children ", Journal of Youth and Adolescence, 18, 39-53.

ANDERSON, J.Z. et G.D. WHITE (1988). "An empirical investigation of interaction and relationship patterns in functional and dysfunctional nuclear families and stepfamilies ", Family Process, 25, 407-422.

BARRY, S. (1988). Le droit de parole de l'enfant dans la séparation parentale. Thèse de doctorat non publiée, Québec, Université Laval.

Barry, S., R. Cloutier, L. Fillion et L. Gosselin (1985). « La place de l'enfant dans les décisions relatives au divorce ", Revue québécoise de psychologie, 6, 86-104. 
BLAIS, M.-C. et R. TESSIER (1990). "L'enfant dans la famille restructurée : Système familial et aspect relationnel ", Apprentissage et socialisation, 13(1), 11-25.

Brand, E. et W.G. Clingempeel (1987). "Interdependencies of marital and stepparent-stepchild relationships and children's psychological adjustment : Research findings and clinical implications ", Family Relations, 36, 140-145.

CAReau, L. et R. Cloutier (1990). " La garde de l'enfant après la séparation. Profil psychosocial et appréciation des familles vivant trois formules différentes ", Apprentissage et socialisation, 13, 55-66.

Clingempeel, W.G. et E. Brand (1985). "Quasi-Kin relationships, structural complexity, and marital quality in stepfamilies: A replication, extension and clinical implications ", Family Relations, 34, 401-409.

Clingempeel, W.G., E. BRand et R. Ievoli (1984). "Stepparent-stepchild relationships in stepmother and stepfather families: A multimethod study », Family Relations, 33, 465-473.

Clingempeel, W.G. et S. SeGal (1986). "Stepparent-stepchild relationships and the psychological adjustment of children in stepmother and stepfather families ", Child Development, 57, 474-484.

Cloutier, R. (1982). Psychologie de l'adolescence, Chicoutimi : Gaëtan Morin.

Cloutier, R. (1985). " L'expérience de l'enfant dans sa famille et son adaptation future ", Apprentissage et socialisation, 8, 87-100.

ClOUTIER, R. (1987). La garde de l'enfant après la séparation des parents. Projet de recherche, École de psychologie, Université Laval, Québec.

Cloutier, R. et G. Groleau (1987). "La communication parents-adolescent ", Interface, 8, 27-30.

Cloutier, R., L. Careau et J. Drolet (1988). La garde partagée : implications psychologiques. Communication présentée dans le cadre du Congrès de la Corporation des psychologues du Québec, Montréal.

Cloutier, R. et A. Renaud (1990). Psychologie de l'enfant, Montréal : Gaëtan Morin.

DEMARIS, A. (1984). "A comparison of remarriages with first marriages on satisfaction in marriage and its relationships to prior cohabitation ", Family Relations, 33, 443-449.

DEMO, D.H. et A.C. ACOCK (1988). " The impact of divorce on children ", Journal of Marriage and the Family, 50, 619-648.

Dolto, F. (1988). La cause des adolescents, Paris : Laffont.

Duberman, L. (1975). The Reconstituted Family, Chicago : Nelson-Hall.

GANONG, L.H. et M. Coleman (1980). "Clinical and empirical literature on stepchildren ", Journal of Marriage and the Family, 48, 309-318.

GILES-SIMS, J. et C. URWIN (1989). "Paternal custody and remarriage », Journal of Divorce, 13, 65-79.

Glick, P. (1984). "Marriage, divorce and living arrangements : Prospective changes ", Journal of Family Issues, 5, 7-26.

Groleau, G. (1987). L'évolution de la communication parent-adolescent-e. Mémoire de maîtrise non publié, Québec, Université Laval.

Hetherington, E.M., M. Stanley-Hagan et E.R. Anderson (1989). " Marital transitions : A child's perspective ", American Psychologist, 44(2), 303-312.

HINDE, R.A. et J. SteVENSON-HINDE (1988). Relationships Within Families, Oxford : Clarendon Press.

Hobart, C.W. (1988). " Perception of parent-child relationship in first married and remarried families", Family Relations, 37, 175-182. 
JESSOP, D.J. (1981). Family relationships as viewed by parents and adolescents : A specification », Journal of Marriage and the Family, 43, 95-108.

KNAUB, P.K., S.L. HanNa et N. Stinnet (1984). "Strengths of remarried families », Journal of Divorce, 7, 41-55.

KURDEK, L.A. (1990). " Effects of child age on the marital quality and psychological distress of newly married mothers and stepfathers ", Journal of Marriage and the Family, 52, 81-85.

Milus, D.M. (1984). "A model for stepfamily development ", Family Relations, 33, 365-372.

Moos, R.H. et B.S. Moos (1986). Family Environment Scale Manual, Palo Alto, Ca. : Consulting Psychologists Press.

Nunnally, J.C. (1967). Psychometric Theory, New York : McGraw-Hill.

OLSON, D.H. et H.I. MCCUBBIN (1983). Families : What Makes Them Work?, Beverly Hills, Ca. : Sage.

Olson, D.H., H.I. McCubbin, H. Barnes, A. Larsen, M. NuXen et M. Wilson (1985). Families Inventories. Family Social Science. University of Minnesota, St. Paul.

Olson, D.H., C.S. RUSSEL et D.H. SPRENKLE (1983). " Circomplex model of marital and family systems : VI theorical update ", Family Process, 22, 69-83.

PAPERNOW, P.L. (1984). "The stepfamily cycle : An experiential model of stepfamily development ", Family Relations, 33, 355-363.

PINK, J.E.T. et K.S. WAMPLER (1985). "Problem areas in stepfamilies : Cohesion, adaptability, and the stepfather-adolescent relationship ", Family Relations, 34, 327-335.

RoberTs, R.N. et P.R. MAGRaB (1991). "Psychologist's role in family-centered approach to practice training and research with young children ", American Psychologist, 46, 144-148.

ROBERTS, T.W. et S.J. PrICE (1989). "Adjustment in remarriage : Communication, cohesion, marital and personal roles ", Journal of Divorce, 13, 17-43.

SAUER, L.W. et M.A. FINE (1988). "Parent-child relationships in stepparent families », Journal of Family Psychology, 1, 434-451.

SMITH, T.E. (1990). "Parental separation and the academic self-concepts of adolescents : An effort to solve the puzzle of separation effects", Journal of Marriage and the Family, 52, 107-118.

SORENSEN, E.R. et J. Goldman (1990). "Custody determinations and child development : A review of the current literature ", Journal of Divorce, 13, 5367.

Statistique Canada (1986). Mariages et divorces. Statistiques de la population, vol. 2 (cat. 84-205), Ottawa, ministère des Approvisionnements et Services.

VISHER, E.B. et J.S. VISHER (1990). " Dynamics of successful stepfamilies ", Journal of Divorce and Remarriage, 14, 3-11.

WAHLER, R.G. et J.E. DUMAS (1989). "Attentional problems in dysfunctional motherchild interactions: An interbehavioral model ", Psychological Bulletin, 105, 116-130.

WHITE, L.K. (1979). "Sex differentials in the effect of remarriage on global happiness ", Journal of Marriage and the Family, 48, 868-876.

WHITE, L.K. et A. BоOTH (1985). "The quality and stability of remarriages : The role of stepchildren ", American Sociological Review, 50, 689-698. 